\title{
Are the Users Own Formal Roles of Authority Most Influently in the Social Mieda? -Based on the Snamethod
}

\author{
Qian Chen ${ }^{1}$
}

WuHan Donghu University, Wuhan,430212,China

${ }^{\mathrm{a}}$ email,

Keywords: New-Farmer, Micro-Blog, Social Network Analysis

\begin{abstract}
The emergency of Chinese new-farmer group has become a widespread phenomenon in the agriculture economy. The most significant characteristic of new-farmer is their embededinterntet gene,that is ,they acquireand speared knowledge, ideas and information through internet, sales agriculture products on various e-commerce platforms, initiate o2o activities frequently. As a result, an online social tie is formed among the new-farmers, which they called it as new-farmer alliance. Based on their social activities on sinamirco-blog, the study examines (1) who is the most influently new-farmer of the alliance in the sinamirco-blog, (2) what is the structure of the social network of the new-farmer alliance in the sinamirco-blog.The second hand data are collected from sinamicro-blog in April 2016, the social network analysis method is used in the study. Finally, the most influent users are founded by using the index of degree centrality, betweenness centrality and closeness centrality, the overall structure of the social network is described by density and cohesive subgroups.
\end{abstract}

\section{Introduction}

The Background of New-Farmer Alliance on Sina Microblog. According to the definition of WIKIPIDEA ,the traditional farmer refers to the person engaged in agriculture,including those engaged in natural economic industry, however, the new farmer refers to the agricultural person living in the countryside or town ,who is euipped with scientific and cultural quality and has a good command of the technology about morden agricultural production, as well as relys on the income of agriculture.compared with the new occupation farmers, the new farmer is a breakthrough.in the research report of Chinese new farmers released by ALI RESEARCH,the internet gene was regarded as the most vital gene of new farmers and they are definited as the product that internet gives the power to three agricultures with being organically combined with them,as well as the representatives of the advanced productivity among the farmer groups.

The union of new farmers is originally the thousands of QQ group named Chinese new famers founded by Simba in 2013. In June ,2013,simba organized the third chinese new farmers helpful salon in the farm village of shenhu, shanghai, discussing the concept and value of new farmers, asking for opinions about founding the union of new farmers and launching a selection activity of SEEKING THE CHINESE NEW FARMERS by sina micro-blog.

The union of new farmers put forward by Simba, the founder of it and Sina micro-blog new farmers conference is a loose organization. the new farmers ,who manufacture the agricultural products and communicate sale by the internet around the nation, focus and support each other .now an enormous group has come into being, which makes the new farmers a social phenomenon. by the end of april 20, 2016, there are 35360 fans in the official homepage microblog farmers conrerence of the sina microblog farmers conference and the microblog account simba in the wild of simba, the founder of new farmers union ,is focused by 16126 fans. the activities of new farmers in the microblog are mainly the following items . 
Firstly, to promote the concept of new farmers and knowledge. the microbiog farmers conference will invite new farmers with a lot of experience in electronic business to give a lecture online regularly . Secondly, to release the information about unsalable agricultural products by microbiog . Thirdly, to launch the activity online and join offline. new farmers organize all the activities offline, discussing the difficult problems ,including informatization production of agriculture, the management of electronic business,t he design of products, logistics, promotion and so on. Finally, to communicate and sale the products. new farmers will release their own products news in the due course by the microblog as well as the discount,to attract the purchase. In addition,new farmers communicate frequently, click with each other and comment ,as well as forward in the microblog. to increase the exposure and interaction between allies.they also strength the relationship between allies by the interaction offline.

The Literature Review of Microblog. Sina microblog is the product promoted by the sina website in july,2009.not only has it become one of the hottest appliances in the filed of internet,but also researched by a lot of scholars in the academia from different angles.nowadays, the following items mainly show the research of micro-blog .in the first place,definite the microblog as a new media,just to research the characters of communication.in the second place, to research the sina microblog with the analytical angle of social internet .microblog internet belongs to scale-free internet,showing the phenomenon of small world.according to the quantity focused from micro-blog users,its scatter observes the power-law distribution,and shows the phenomenon of long-tailed distribution. releasing the document by users also seemingly presents the power-law distribution. in the third place, among the most of users ,the links are found one-way in the research of micro-blog users. in the same time ,there are core users in the sina micro-blog.

The Research Question of the Study. The paper attempts to make use of the methods of analysing the social internet,to research whether core users online are relevant with those offline among the members of the new farmers union,registering the sina micro-blog, and how the character of internet structure of the new farmers is ,in the sina micro-blog. datas used by the essay are from the top 50 members,in the influential ranking list concerning the agricultural micro-blogs of big $\mathrm{v}$,release by the micro-blog farmers conference in 2016,april.we can find the character online of social internet between core users online and members ,by the mutual relationship of 50 members.

\section{The Introduction of SNA(Social Network Analysis)}

The Perspective, Hypothesis and Content of SNA. Social network analysis uses structure-relation viewing angle, which means actors participate in social system, and this system relates to actors and the behaviors of others. The relation of actors and social structure have an important influence on the behaviors of actors. Therefore, the kernel of social network analysis theory is relation-structure. Date of tie is the foundation of social network theory, model and application.(social)Network analysis about relational model and the utility of relational model include following hypotheses:

For comprehending observed behaviors, the structural relation is more important than individual feature such as age, gender, value, awareness and action etc.

Social network influences hypostatic concept, belief and action by various architectural mechanisms which established by entities.

A structure-relation should be regard as a kind of dynamic process. SNA method could measure individual actor and the complex relation and junction tie between social network members

(Wellman, 1997), and this method could make visual modeling for the communication mode of group members (Monge\&Contractor, 2001).SNA method also could promote the comprehension 
of social network structure such as Internet and so on（Park，2003) .The validity of SNA method is that this method can lead us to perspectively see the interaction between actors in social network. SNA method could define and explain this kind of interaction, and see schemata with mutual connection created by actors to comprehend the significance of this schemata (Haythornthwaite, 2005).

The Three Analysis Unit of SNA. SNA includes three analytical units: Actors, which are the node of network, are the members or events in fictitious study community. Relationship, which is the line between nodes in network, reflect the interaction between actors and various kind of social relationship shaped by this interaction. Tie, which is the set of a kind of relationship, have a range from weak to strong.

The Relationship, Types and Tools of the SNA Examined. The relationship which SNA researches, includes content, direction and strength. The content of relationship involves what actors share and exchange; The direction of relationship involves whor emit relationship and who is directed; The strength of relationship involves the quantity and frequency of exchanged information between actors (Haythornthwaite, 2005). The direction of relationship could divide into undirected relationship and directed relationship. Undirected relationship normally focuses on whether the relationship exist and directed relationship focuses on whether actor is the accepter or sponsor of relationship.

According the collecting method of data, SNA has two analysis types: Ego-CenteredNetworks analysis andWholeNetworks analysis (Borgatti, 1998).Ego-CenteredNetworks analysis regard special actors as researchemphasis and center, and it only consider the relationship set related to actors of emphasis.. Using this type of analysis, researcher should focus on a actor. The content of research has the size, difference, property and so on ofEgo-CenteredNetworks. WholeNetworks analysis research the relationship of all actors in a special range,

Sociogram and Social Matrix are not only the carrier of the social network data storage ,but also the most commonly used analysis tool in SNA.Sociogram shows relation mode, consists of the point of represent of the actors and the line of represent of the relation between actors.InSociogram, if the line without direction is undirected graph, otherwise as a directed graph.If you give the line to a certain value in the graph, used the value to represent of the strength of the relation, is called valued graph. Use of the advantages of Sociogram is directly expresses relation network, and when the number of actors becomes more in graph, the graph becomes very complicated and difficult to visually analyze relational structure. At this time, Social Matrix shows its special role. Social Matrix also called Adjacency Matrix, rows and columns represent the same actors , and the order is same. The rows representrelational sender but the columns represent relational receiver; If use 0 s and 1 s represent the value of adjacency matrix, it called binary matrix; If expressed in a specific figure, it called the assignment matrix.The advantages of Sociogram can represent a larger complex networks, and rearrangement, transpose, addition and subtraction, multiplication and power, inverse, correlation and regression arithmetic in matrix can be used respectively to analyze network of Cohesive Subgroup, Cliques andBlock model, etc. (Liu Jun, 2004). Because SNA requires a lot of matrix operations, SNA computer aided software is also the most commonly used SNA analysis tool.Now, Computer aided software is used to support the SNA probably already have 27 kinds, commonly used have UCINET, Pajek, NetMiner, STRUCTURE, MultiNet and StOCNET, etc.

\section{How to Find the Most Influent New-Farmer on Sinamicroblog}

The Indexes of Core Users Determination. The popularity is used to confirm the core users in 
sina microblog. popularity analysis method consider the choice and be choose under econometrics and the construct and model of indirect relationship. Individual popularity reflects his visibility among crowd. group popularity index estimate the distraction and unbalance degree in a network. in this paper, the individual popularity is used in measuring the core new famer user of sina microblog. specifically, the measuring method of popularity are centrality and prestige. Centrality can be divided to point centrality, local centrality and global centrality. local centrality refers to the relative importance of the local point to its neighbor point, while the global centrality means the strategy importance of the point in the whole network. the most widespread used centrality includes degree centrality, closeness centrality, betweenness centrality and eigenvector centrality.

Data Collection and Coding. This paper intend to use the data of new famer big VIP list which published by the microblog famer chamber in april,2016. The microblog famer chamber publishes the list every bio-month, the selected standard is according to the influence of the users, which calculated by the added value of activity, transmissibility, coverage. the activity measurement depends on the effective items the users post, relay and review initiatively. transmissibility measurement according to the effective items the users' post be relayed and reviewed. coverage is measured by the active fans of the uers. based on the data collected, we can express the relationship of the users by a adjacency matrix, the matrix row represents the follower while the matrix column stands for the users be followed. if the follow relationship exists, 1 is assignment, otherwise the 0 is filled in the matrix.

\section{Methodology}

Determining newfamer's core users in micro-blog by way of popularity. Popularity analysis is to consider the structure and pattern of choice and being choiced, as well as indirect relationships.Individual actor's popularity obviously reflects its extent of visibility among other actors. Otherwise, popularity indexes are used to estimate the popularity's extent of dispersions and imbalances for all actors in a network.In this article, the determination of new-famer core users on micro-blog is measured from the angle of individual actors' popularity. In social networks, the index to measure popularity mainly comprises centrality and prestige. What centrality mean is that no matter as a relationship's initiator or a receiver, when one enjoys great popularity, he has high level of participation as well to multiple relationships .Prestige means that even if actors with great popularity launch less relationship, he'll receive many direct relationships.

Centrality consists of three parts: point centrality, local centrality and global centrality.Point centrality explores every point's relative centrality in the social network diagram. Lo cal centrality refers to the relative significance of a local centrality to its neighboring points. And th e global centrality refers to the strategic importance of the point in the overall network.In the social network, the most widely used center measurement standards include degree centrality, closeness centrality, between nesscentrality and eigenvector centrality. In anundirected binary graph, anactor's degree centrality measures the degree to which a node is connected to all other nodes in the networks. The closeness centrality is used to reflect the degree of closeness between a node and the other nodes in socialnetworks. In an undirected binary graph, what actors'betweenness centrality measures is that to what extent a node located in the middle of the other nodes in the graph , namely anactor becomes a broker or a middleman, or to what extent control other actors. The betweenness centrality's focus is on how actors control and regulate the relationships between two nodes not directly related to, it's a critical index about controlling the exchange of information and the flowing of resources. And the eigenvector centrality means anactor with high eigenvector centrality, who establishes linkages with him will be connected by many other actors. 


\section{Result}

The Degree Centrality. Entering the matrix to the UCINET software can calculatethe degree centrality. you can calculate the top 100 new-famers' every out-degree and in-degree in micro-blog. In-degree is the extent to the relationship directed, namely to pay attention to other users. From the result we can see that which users'in-degreeis the highest and it means the information they have released is observed by more people and has great power in whole networks as well.

The betwenness Centrality. Entering the same focus matrix to the UNINET software can calculatebetweenness centrality and its central potential. As reflected in figure 8, we can calculatethe top 100 sina micro-blog users of each new-famer's statistic to betweenness centrality.Each node's betweenness centrality measures the extent of information's flow or spread's control function in whole networks, that is to say to what extent as node A is conveyed to node B would rely on node $\mathrm{C}$. Analysis result shows that the users with high betweennesscentrality , so the power of these several users is quite a bit, besides they can control the flow of information to a large extent. It may also indicate the betweenness center potencial from the results. When the center potencial is high, it signifies most of nodes need other nodes to be the bridge nodes to get information in the whole networks.

The Overall Social Structural of New-Farmer Alliance on Sina Micro-Blog. When the relationship between some actors in the network is too close to be united as a secondary group,in society network analyze,this kind of group is called Cohesive Subgroups. A Cohesive Subgroups Analysis is to study,how many Subgroups like this exist in the network; the characteristics of insiders in the Subgroup;the characteristics between Subgroups; the characteristic of the connection between the member from a Subgroup and one from another.Specifically speaking,Cohesive Subgroups Analysis is an actor subset meeting the following needs, in which subset the actors show better 、 directer、 closer、more often or more active relationship. A Cohesive Subgroup should be a group which links closely to its inside,in a specific research,we should focus on the character inside the Subgroup and analyze the characteristics between insides and outsides. As far as relevance is concerned,a Cohesive Subgroup should be relatively stable,which means that a Cohesive Subgroup would not become unrelated for being out of several members of it. To speak more specifically,when taking away some lines from it,a coupe of points can still be connected with each other. We can make quantitative measurement with "line connectivity" by this couple of points. We assume these two points as $i$ and $j$,the line connectivity as $\lambda(i, j)$, which makes any approaches between $i$ and $j$ not exist and the minimum line must be wiped off. The smaller the $\lambda(i, j)$ is, the more sensitive $\mathrm{i}$ and $\mathrm{j}$ is to the wiped-off lines,which means they seem more likely to separate.Otherwise, the bigger the $\lambda(\mathrm{i}, \mathrm{j})$ is, the less sensitive $\mathrm{i}$ and $\mathrm{j}$ is,that's to say,to separate $\mathrm{i}$ and j,more lines should be wiped off from the Subgroup. More specifically to say,the bigger the $\lambda(i, j)$ is,the more stable they are;the smaller the $\lambda(i, j)$ is, the more sensitive they are.Based on the different theories and methods of measurement, it exists different types of definition and analyze ways among Cohesive Subgroups.

\section{Acknowledgements}

Fund Project: This work was supported by the grants from "The Research of Agricultural information service system construction to promote agricultural industrialization management " of Wuhan Donghu UniversityAgriculture EC Hubei Provincial Collaborative Innovation Ccenter.

\section{Reference}


[1] Guo Z, Li Z, Tu H. SinaMicroblog: An Information-Driven Online Social Network[C] International Conference on Cyberworlds, $\mathrm{Cw}$ 2011, Calgary, Alberta, Canada, October. 2011:160-167.

[2]Pengyi Fan, Pei Li, Zhihong Jiang, et al. Measurement and analysis of topology and information propagation on Sina-Microblog[C] Intelligence and Security Informatics (ISI), 2011 IEEE International Conference on. IEEE, 2011:396-401.

[3] Liu J, Cao Z, Cui K, et al. Identifying Important Users in SinaMicroblog[C] Fourth International Conference on Multimedia Information NETWORKING and Security. IEEE Computer Society, 2012:839-842.

[4]Wang C, Guan X, Qin T, et al. Modeling the heterogeneity of human dynamics based on the measurements of influential users in SinaMicroblog[J]. Physica A Statistical Mechanics \& Its Applications, 2015, 428:239-249. 